\title{
Wilms' Tumor: Long-Term Effect of Multimodal Treatment on Renal Function
}

Michelle S. McLauchlin (1), B.Sc., Elizabeth Cairney (2), M.D., Barry deVeber (2), M.D., R. Morrison Hurley (2), M.D., and Douglas G. Matsell $(2,3)<$, M.D. br>

To whom correspondence should be addressed:

1. Department of Nephrology

The Children's Hospital of Western Ontario, London, ON, Canada N6A 4G5

2. Department of Paediatrics, The Children's Hospital of Western Ontario

3. Child Health Research Institute, University of Western Ontario

\begin{abstract}
Patients with Wilms' tumor, a common malignant childhood tumor, have an excellent survival rate. The treatment protocol, which consists of a combination of surgery, chemotherapy, and irradiation, may induce both short- and long-term systemic effects. This paper addresses concerns regarding the effects of treatment on long-term renal function in the survivors of Wilms' tumor. In a retrospective study, the charts of 36 patients treated at the Children's Hospital of Western Ontario were analyzed with respect to the epidemiology and parameters of renal function. It was determined that patients with Stage I disease maintained normal renal function over five years. However, those with Stage III and IV disease exhibited decreased renal function immediately after treatment but regained normal renal function within five years. This investigation clearly establishes the need for future prospective studies of long-term renal function of the remaining kidney in children who have undergone treatment for Wilms' tumor.
\end{abstract}

\section{INTRODUCTION}

Wilms' tumor (WT) is the most common abdominal tumor in children. Globally, the incidence of this childhood cancer is approximately 1 in 10,000 children (1). Tumors typically present in children under the age of 15 years, most commonly between the ages of 3 and 5 years.

WT is a malignant embryonal tumor that develops from the rapid proliferation of renal metanephric blastema. In most cases, an asymptomatic abdominal mass is discovered by the parent or by the physician during routine physical examination. However, abdominal pain, hematuria, hypertension, fever, malaise, and weight loss may also be present (2).

Both hereditary and sporadic forms of the disease exist. Several different genes may be involved, one of which has been located on the short arm of chromosome 11 (band 11p13) (3). Aniridia, hemihypertrophy, and Beckwith-Wiedemann syndrome are often associated with WT, making screening of patients with these signs 
a priority. Bilateral tumors are more likely to result from the hereditary form and occur in $5-10 \%$ of WT patients (4). These children have a worse prognosis than those afflicted with the more common unilateral WT.

Other prognostic indicators of WT include the stage at diagnosis, the histopathologic features of the tumor, and the age of patient. Tumor staging has been devised by the National Wilms' Tumor Study Group. Stage I is limited to the kidney within the capsule and can be completely excised through surgery. Stage II extends beyond the kidney capsule and can also be completely excised. Patients with Stage III disease have tumor metastases confined to the abdomen, while those with Stage IV disease have hematogenously disseminated metastases, predominantly to the liver and lungs. Stage V refers to bilateral renal involvement at the time of diagnosis (5).

Therapy for WT consists of surgery (i.e., uninephrectomy), postoperative and more recently preoperative chemotherapy (some combination of vincristine, actinomycin D, adriamycin, etoposide, cyclophosphamide, or cisplatin), and in some cases, irradiation (6). Over the past two decades, more effective protocols for the treatment of WT consisting of chemotherapy, irradiation, and surgical techniques have been developed. The primary goal has been to reduce the predictable adverse consequences of current short- and long-term treatment modalities. The use of toxic, high-dose chemotherapeutic agents has been reduced and radiotherapy is presently reserved for higher-stage disease. The improvements in treatment protocol and earlier, more accurate detection of WT have resulted in a current five-year survival rate of $90 \%$ for all stages combined (7).

Long-term sequelae following multimodal treatment protocols have been described by several authors. Complications include secondary malignant neoplasms such as leukemia, lymphoma, sarcoma, and carcinoma, as well as cardiac abnormalities, growth, and developmental delay (5). In addition, specific effects on renal function have been noted. The chemotherapeutic agent, ifosfamide, causes tubular nephropathy and can result in a complete Fanconi syndrome $(8,9)$. Cisplatin may also lead to unpredictable and severe renal damage, especially when combined with other nephrotoxic agents such as aminoglycoside antibiotics (10). Furthermore, the use of actinomycin D with other chemotherapeutic agents worsens renal damage when used with irradiation (11).

The long-term effects of treatment on renal function have not been clearly defined. However, progressive renal dysfunction in WT patients may result from the removal of functional renal tissue through surgical nephrectomy and acute or chronic damage caused by chemotherapy. Uninephrectomy alone results in few, if any, adverse consequences over a period of 10 to 20 years $(12,13)$. We therefore studied the effects of the combination of chemotherapy, irradiation, and uninephrectomy on long-term renal function in children with WT.

The purpose of this retrospective study was to determine the effect of multimodal therapy on renal function up to five years following completion of treatment. The epidemiology and clinical presentation of WT in Southwestern Ontario over the past 20 years are also described.

\section{METHODS}

A retrospective study analyzing the charts of 36 patients diagnosed with unilateral WT from 1974 to August of 1994 was performed. All patients were treated at the Children's Hospital of Western Ontario in London. The treatment protocol was documented and found to be relatively consistent within each stage (Table 1).

Serial measurements of serum creatinine, proteinuria, and hematuria were recorded from the patients' charts. In addition, the patients' gender, place of birth, and age at the time of diagnosis were noted. The staging and clinical description of the tumor, along with associated signs, symptoms, and relapse interval were recorded. The cases were subsequently grouped according to stage at the time of diagnosis. 
The renal function of patients with Stage I, III, and IV disease was estimated through standardization of the serum creatinine levels. Insufficient data were available for Stage II patients diagnosed during the period of study. Therefore, renal function for this group was not included. Each individual serum creatinine level was divided by the mean value for age and gender (14). As renal function is inversely related to creatinine levels, the inverse of the above calculation was determined, multiplied by 100, and expressed as a percent. The resultant value offers a reflection of the renal function. Serum creatinine measurement is perhaps the simplest assessment of renal function and is a relative indicator of the glomerular filtration rate (GFR) (15).

The data were entered into Microsoft Excel for Macintosh (Redmond, WA, USA) and were then plotted over a five-year period. Stage III and IV patients shared similar treatment protocols and were grouped together for statistical analysis. The differences between Stage I and Stages III and IV together at each time point were analyzed using the Student's t test (one tail). A difference was considered statistically significant if $\mathrm{p}<0.05$. Statistical analysis was performed using the Macintosh program Statview 512 (Calabasas, CA, USA).

\section{RESULTS}

\section{Epidemiology}

The patients referred with WT were distributed throughout Southwestern Ontario (Fig. 1), and the number of patients referred was proportional to the population of the referring center; therefore, there did not appear to be any regional differences in the incidence of the disease.

\section{Pathophysiology}

Histopathology may take the form of favorable or unfavorable cellular patterns. The unfavorable histology (UH) is further subdivided into anaplastic (16) and sarcomatous (17) subtypes. UH occurs in $10 \%$ of patients and carries a much worse prognosis than the favorable histology $(\mathrm{FH})$.

Our patient sample consisted of 18 Stage I, 3 Stage II, 8 Stage III (6 with FH, 2 with UH), and 7 Stage IV (4 with FH, 3 with $\mathrm{UH}$ ) children (Table 2). The age at the time of diagnosis varied from one month to 16 years. Stage I, II, III, and IV were diagnosed at $4.3+/-3.6,5.0+/-1.0,5.4+/-3.2$, and $8.0+/-4.3$ years respectively. The male to female gender ratios $(\mathrm{M}: \mathrm{F})$ for each stage were noted and are presented in Table 2 . Two children with Stage IV disease died, one within a year after treatment, and the other within three years. These patients were included in the data.

\section{Presenting Signs and Symptoms}

The majority of patients (60\%) with Wilms' tumor presented with an asymptomatic abdominal mass (Fig. 2). Of these, the majority were asymptomatic, while some also had some abdominal pain (11.1\%) or fatigue $(5.6 \%)$. As a presenting feature, hematuria occurred by itself in $6 \%$ of the patients and in association with abdominal pain in $11 \%$. Rare presentations included bowel obstruction $(2.8 \%)$, pyelonephritis $(2.8 \%)$, hypertension $(2.8 \%)$, and heart failure (2.8\%). Neither aniridia nor hemihypertrophy was documented in any of the patients.

\section{Renal Function}

Renal function was determined following the completion of treatment. Serum creatinine levels standardized for age and gender revealed that Stage I patients maintained normal function throughout the five year study period (Fig. 3). As mentioned in Methods, renal function values for patients with Stage II disease were not 
included due to insufficient data. Patients with Stage III and IV disease, when combined, had significantly poorer renal function than patients with Stage I disease over the first three years after treatment, as reflected by the standardized serum creatinine. This difference was statistically significant when analyzed at $0.5,1$, and 2 years. Over five years following the completion of treatment, normal renal function was maintained by Stage I patients and was achieved by Stage III and IV patients.

\section{DISCUSSION}

With the improved survival of children treated for malignancy, long-term side effects of treatment are becoming increasingly recognized. It is important to monitor long-term renal function in these children considering the excellent survival rate of patients with WT, the toxicity of chemotherapy, and the demands of growth on a single kidney.

The results of this study demonstrate that children with Stage I disease, who were treated primarily with chemotherapy and uninephrectomy, maintained normal renal function during the five years of follow-up. This suggests that adequate renal tissue is preserved and that the effects of treatment may be negligible as late as five years after treatment.

However, children with Stage III and Stage IV WT, who were treated with uninephrectomy, chemotherapy, and often irradiation, had impaired renal function soon after treatment. The causes of this dysfunction are likely mutifactorial and are related to the exposure to chemotherapy and other nephrotoxins. An alteration in renal function, as reflected by an abnormal serum creatinine, tubular proteinuria, or hypertension, has been previously recognized (18-21).

These patients' early impaired renal function eventually appeared to normalize within the five-year period after treatment; this is consistent with the findings of Makipernaa et al. who reported that survivors of WT seldom had long-term renal sequelae or other systemic complications (22). Our observation suggests that renal function is normalized by the remaining kidney, possibly as a result of compensatory hypertrophy and/or recovery of normal renal parenchyma following the nephrotoxicity of chemotherapy.

Although our patients recovered normal renal function within five years, two very ill patients with Stage IV WT and poor renal function died within the period of analysis. This effect may have lowered the initial posttreatment renal function mean value and accentuated the rise of renal function of the group toward 100\% of normal. Two patients with poor renal function who were studied with nuclear glomerular filtration rate (GFR) investigations demonstrated a consistent decline in renal function at long-term follow-up (data not shown).

As in any retrospective study, many variables may have affected our results. We were unable to precisely control for the treatment protocols as they changed in subtle ways throughout the 20 years. Documentation of renal function indices, including serum creatinine, blood pressure, and urinalyses was not standardized and was at times sporadic.

In this study, we describe the regional distribution and epidemiology of the childhood Wilms' tumor in Southwestern Ontario over the past 20 years. In addition, we describe renal function in these patients up to five years of follow-up after the completion of treatment. The results demonstrate altered renal function soon after treatment in children with higher stages of disease but not after five years. While there appears to be an improvement in function over time, this trend is confounded by patient dropout due to death. Nonetheless, these results suggest the need for further prospective studies of renal function in long-term survivors of Wilms' tumor, specifically monitoring blood pressure, proteinuria, creatinine clearance, and glomerular filtration rate.

\section{ACKNOWLEDGEMENTS}


We would like to thank Dr. K. W. Speechley for her advice concerning data collection, and Ms. Elizabeth Millar for her expert assistance in the preparation of this manuscript. This work was supported by a grant from the Chapter of Odd Fellows and Rebeccas (MSM), and in part by the Child Health Research Institute (DGM).

\section{REFERENCES}

1. Young JL, Ries LG, Silverberg E, Horm JW, Miller RW. Cancer incidence, survival, and mortality for children younger than 15 years. Cancer 1986;58:598-602.

2. Oski FA. Principles and Practice of Pediatrics. J.B. Lippincott Company, 1990:1587-1589.

3. Haber DA, Buckler AJ, Glaser T, et al. An internal deletion within an 11 p13 zinc finger gene contributes to the development of Wilms' tumor. Cell 1990;461(7):1257-1269.

4. Coppes MJ, de Kraker J, van Dijken PJ, et al. Bilateral Wilms' tumor: long-term survival and some epidemiological features. Journal of Clinical Oncology 1989;7(3):310-315.

5. National Wilms' tumor Study Committee. Wilms' tumor: status report, 1990. Journal of Clinical Oncology 1991;9(5):877-887.

6. D'Angio GJ, Breslow N, Beckwith JB, et al. Treatment of Wilms' tumor: results of the third National Wilms' tumor Study. Cancer 1989;64(2):349-360.

7. D'Angio GJ, Evans A, Breslow N, et al. The treatment of Wilms' tumor: results of the second national Wilms' tumor Study. Cancer 1981;47:2302-11.

8. Willemse PHB, de Jong PE, Elema JD, Mulder NH. Severe renal failure following high-dose ifosfamide and mesna. Cancer Chemotherapy and Pharmacology 1989;23:329-330.

9. Moncrieff M, Foot A. Fanconi syndrome after ifosfamide. Cancer Chemotherapy and Pharmacology 1989;23:121-122.

10. Womer RB, Pritchard J, Barratt TM. Renal Toxicity of cisplatin in children. Journal of Pediatrics 1985;106:659.

11. Goldberg ID, Garnick MB, Bloomer WD. Urinary tract toxic effects of cancer therapy. The Journal of Urology 1984;132(1):1-6.

12. Narkun-Burgess DM, Nolan CR, Norman, JE, Page WF, Miller PL, Meyer TW. Forty-five year followup after uninephrectomy. Kidney International 1993;43:1110-1115.

13. Robitaille P, Mongeau JG, Lortie L, Sinnassamy P. Long-term follow-up of patients who underwent unilateral nephrectomy in childhood. Lancet 1985;1:1297-1299.

14. Schwartz GJ, Haycock GB, Spitzer A. Plasma creatinine and urea concentration in children: normal values for age and sex. The Journal of Pediatrics 1976;88:828.

15. Holliday MA, Barratt TM, Avner ED. Pediatric Nephrology. 3rd ed. Williams \& Wilkins, 1994:74-75.

16. Zuppan CW, Beckwith JB, Luckey DW. Anaplasia in unilateral Wilms' tumor: a report from the National Wilms' tumor Study Pathology Center. Human Pathology 1988;19(10):1199-1209. 
17. Haas JE, Bonadio JF, Beckwith JB. Clear cell sarcoma of the kidney with emphasis on ultrastructural studies. Cancer 1984;54(12):2978-2987.

18. de Graaf SSN, van Luyk WHJ, Tamminga RYJ, Mehta DM. Prospective study of renal function in Wilms' tumor patients. Abstract. May, 1990.

19. Barrera M, Roy LP, Stevens N. Long-term follow-up after unilateral nephrectomy and radiotherapy for Wilms' tumor. Pediatric Nephrology 1989;3:430-432.

20. Mitus A, Tefft M, Fellers FX. Long-term follow-up of renal functions of 108 children who underwent nephrectomy for malignant disease. Pediatrics 1969;44:912-921.

21. Thomas PR, Griffith KD, Fineberg BB, et al. Late effects of treatment for Wilms' tumor. International Journal of Radiation Oncology, Biology, Physics 1983;9:651-657.

22. Makipernaa A, Koskimies O, Jaaskelainen J, Teppo AM, Siimes MA. Renal growth and function 11-28 yrs after treatment of Wilms' tumor. European Journal of Pediatrics 1991;150:444-447.

\section{AUTHOR BIOGRAPHY}

Michelle S. McLauchlin received a B.Sc. degree in Biology from the University of Western Ontario (London, Ontario) in 1992. She is presently a third-year medical student at the University of Western Ontario. Her work on Wilms' tumor was conducted during her second year of medical education at the Children's Hospital of Western Ontario. This research was supported by grants from the Chapter of Odd Fellows and Rebeccas (MSM) and the Child Health Research Institute (DGM). 